\title{
The Drosophila Pax6 paralogs have different functions in head development but can partially substitute for each other
}

\author{
Linn Jacobsson • Jesper Kronhamn • \\ Åsa Rasmuson-Lestander
}

Received: 10 April 2008 / Accepted: 8 May 2009 / Published online: 30 May 2009

(C) The Author(s) 2009. This article is published with open access at Springerlink.com

\begin{abstract}
There are two Pax6 genes in Drosophila melanogaster; eyeless (ey) and twin-of-eyeless (toy), due to a duplication, which most likely occurred in the insect lineage. They encode transcription factors important for head development. Misexpression of either toy or ey can induce formation of ectopic compound eyes. Toy regulates the $e y$ gene by binding to an eye-specific enhancer in its second intron. However, Toy can induce ectopic eyes also in an $e y^{-}$background, which indicates a redundancy between the two Pax6 copies in eye formation. To elucidate to what extent these two genes are interchangeable, we first generated toy-Gal4 constructs capable of driving the Pax6 genes in a toy-specific manner. Genetic dissection of the promoter proximal region of toy identified a 1,300-bp region around the canonical transcription start that is sufficient to drive toy expression in embryonic brain and eye primorida and in larval eye-antennal discs. We find that exogenous expression of toy can partially rescue the lethality and eye phenotype caused by lethal mutations in ey and vice versa. We therefore conclude that Toy and Ey, to some extent, can
\end{abstract}

Communicated by T. Clandinin.

L. Jacobsson and J. Kronhamn contributed equally to this work.

Electronic supplementary material The online version of this article (doi:10.1007/s00438-009-0458-2) contains supplementary material, which is available to authorized users.

L. Jacobsson · Å. Rasmuson-Lestander ( $\bowtie)$

Department of Molecular Biology,

Umeå University, 90187 Umeå, Sweden

e-mail: rasmuson@molbiol.umu.se

J. Kronhamn

Umeå Centre for Molecular Pathogenesis,

Umeå University, 90187 Umeå, Sweden substitute for each other. Nevertheless, the phenotypes of the rescued flies indicate that the two Pax6 genes are specialized to regulate defined structures of the fly head.

Keywords Pax6 $\cdot$ Twin-of-eyeless $\cdot$ Eyeless .

Mutant rescue $\cdot$ Drosophila

\section{Introduction}

The Drosophila body plan is established in the early embryo by gradients of maternally deposited mRNA and proteins. A positional coordinate system is formed by combinatorial genetic interactions between gene activators and repressors, subdividing the embryo into discrete domains. Specific cephalic gap genes, like ocelliless, buttonhead, empty spiracles and tailless, define the developmental pattern in the pregnathal region of the head (Finkelstein and Perrimon 1991). The different organs and appendages are then determined by downstream selector genes. At the cellular blastoderm stage, 6-23 cells are allocated to form the eye-antennal primordium (Callaerts et al. 2006). At the beginning of larval development there are about 20 cells, derived from multiple embryonic segments that proliferate to form a larval eye-antennal imaginal disc (Garcia-Bellido and Merriam 1969). After differentiation, this pair of discs will form most of the head capsule of the adult fly.

Pax genes play important roles in the development of the central nervous system (CNS) and brain, as well as of the peripheral nervous system and sensory organs (Chi and Epstein 2002). They encode transcription factors characterized by a 128-amino acid paired domain, which has a sequence-specific DNA-binding function. Pax genes have been divided into five subgroups based on sequence similarity between their paired domains (Breitling and Gerber 
2000; Miller et al. 2000). Several Pax proteins, including Pax6, possess an additional DNA-binding domain, a homeodomain. In Drosophila, the two Pax6 genes: twin-ofeyeless (toy) and eyeless (ey), and the two Pax6-like genes: eye gone (eyg) and twin of eye gone (toe) are all expressed in the same developmental pathway controlling eye development (Halder et al. 1995; Czerny et al. 1999; Jang et al. 2003). How these proteins cooperate to specify the visual system is still under investigation.

In situ hybridizations have shown that toy transcription is initiated at cellular blastoderm in the procephalic neuroectoderm, which gives rise to the primordia of the visual system and the brain (Czerny et al. 1999; Kammermeier et al. 2001). During gastrulation the expression is confined to the region anterior to the cephalic furrow and at germ band retraction toy mRNA is detected in the eye and brain primordia and in a reiterated segmental pattern in the ventral nerve cord (VNC), partially overlapping Ey expression (Kammermeier et al. 2001). In larvae, toy is expressed in certain parts of the brain and VNC, not overlapping with ey: however, in undifferentiated eye discs the pattern is similar to $e y$. Thus, toy and ey have overlapping expression in the developing visual system during Drosophila embryonic development and in the eye-antennal imaginal discs, but non-overlapping in CNS (Kammermeier et al. 2001).

Biochemical data indicate that Toy directly regulates $e y$ by binding to the eye-specific enhancer located in the second intron of the ey gene (Czerny et al. 1999). Mutations in Toy-binding sites within the minimal ey enhancer abolish reporter gene expression in the eye disc primordia (Hauck et al. 1999), but do not entirely eliminate ey expression in the posterior region of the eye discs or in the CNS, indicating that $e y$ is also activated by other means. toy mRNA is present in the eye primordia of $e y^{2}$ and $e y^{R}$ embryos (Czerny et al. 1999) and Punzo et al. (2004) showed that Toy can activate eye development also in an ey-independent manner, probably through its direct binding to the eye enhancer of sine oculis (so). Further on, Ey and So bind to cis-regulatory elements in the atonal gene, and thus directly control neurogenesis in the eye (Zhang et al. 2006). While null alleles of toy result in a headless phenotype (Kronhamn et al. 2002), the hypomorphic alleles $e y^{2}$ or $e y^{R}$ only affect the compound eye development, as does the ey stated null mutant $e y^{J 5.71}$ (Punzo et al. 2004). We have previously described another $e y$ allele, $e y^{D}$, which exhibits a dominant small-eyed phenotype and a recessive headless phenotype, similar to toy ${ }^{\text {hdl }}$ (Kronhamn et al. 2002). Since hemizygous $e y^{-}$flies $(D f(4) B A /+)$ have a wild-type eye phenotype, $e y^{D}$ cannot be considered to be a null mutant. It is caused by an insertion of material from chromosome 2 in the $3^{\prime}$ region of the gene, resulting in a truncated Ey protein which lacks the homeodomain. This protein probably has a dominant negative function, interfering with the wild-type Ey protein.
There is extensive cell death occurring in $e y^{D}$ eye discs, and the phenotype can be rescued by targeted expression of an apoptosis inhibitor (Kronhamn et al. 2002). Since both ey and toy can give rise to ectopic eyes when misexpressed (Halder et al. 1995; Czerny et al. 1999) and since mutants in both genes can produce similar headless phenotypes (Kronhamn et al. 2002), we decided to investigate to what extent these two genes can substitute for each other.

We started out by constructing toy-Gal4 driver lines to be able to express genes in a toy-specific pattern. This was done by dissecting the $5^{\prime}$ upstream cis-regulatory region of the toy gene. We identified a 1,300-bp region surrounding the toy transcription start which was sufficient to drive reporter genes with the expected expression pattern in embryonic eye-antennal primordia and brain as well as in larval eye-antennal discs. The same region was also found to generate headless pharate adults, when driving the expression of an RNAi transgene, which interferes with the translational machinery. This phenotype is similar to the one produced by toy null mutants.

To eventually test possible redundancy between the toy and ey genes we set out to rescue the phenotypes of some lethal alleles in both genes, using the GAL4/UAS system (Brand and Perrimon 1994). To this end we used transgenic lines expressing Gal4 directed by either the toy 5' regulatory region generated or the eye-specific enhancer from the ey gene (Bonini et al. 1997; Hauck et al. 1999) to drive UAS-Pax6 genes and analyzed the viability and head phenotype of the rescued flies. We found that exogenous Toy could significantly rescue both toy and ey homozygous mutants to viability. Correspondingly, the pupal lethality of toy ${ }^{\text {hdl }}$ and toy ${ }^{G 7.39}$ mutants was rescued by expression of Ey. Partial rescue of the gene-specific eye phenotypes was also evident. Furthermore, we show that inhibition of apoptosis increased survival of toy hdl homozygotes, but did not improve eye phenotypes.

\section{Materials and methods}

\section{GAL4 constructs}

In order to study the regulation of the toy gene and to construct Gal4 driver lines with toy $5^{\prime}$ cis-regulatory regions, six different genomic fragments from the region upstream of the toy transcription start were PCR amplified. We used the following primers: AGCTGGGATCCTATGGGATAT GTATATATG (used for constructs toyl and toy5); AGG CAAGGCATTTTGAACTCCTGGTACCGT (toy2); AAT GGTACCGGGCAATAAATAACTAGTC (toy3); GAAT CCATAATGTTAAAGGTACCCACGAT (toy4); TATG CACTAAGCAAATGGTACCGAAAGAG (toy); TTAG GATCCTAATTAATTATTTTTAATGCG (toyl, toy2, 
toy3, toy4); ACGGATCCAGGAAGGTCAAACAGTA TCA (toy5, toy6). (For extension and overlap between fragments see Fig. 3.)

The fragments toy2, toy3, toy4, and toy6 were first cloned into the $p G a T B$ vector. They were then cut out together with the Gal4 gene, using NotI and KpnI, and ligated into a $p P\{C a S p e R-4\}$ vector. For subcloning of toy 1 and toy5, the Gal4::Hsp70Bb portion of $p G a T B$ was first cut out using KpnI and SstII and then ligated into the polycloning site of $p P\{C a S p e R-4\}$. To generate a unique BamHI site upstream of the Gal4 coding region, the BamHI in the polycloning site was removed by cutting with NotI and StuI, treating with nuclease S1 to generate blunt ends and re-ligating. Fragments toy 1 and toy 5 were then ligated into the remaining BamHI site and clones were sequenced to confirm correct orientation. The constructs were transformed into $w^{1118}$ embryos. Several independent inserts were generated for each construct. The number of analyzed lines is shown in Supplementary Table S1.

Toy antibody production, Western blot and immunostaining

Polyclonal antiserum directed against Toy was raised in rabbit (Agrisera, Vännäs, Sweden). Two Toy peptides that differ significantly from Ey, were simultaneously used as antigens: amino acids 209-224 and 531-543. The antiserum was affinity purified using an UltraLink matrix (Pierce) with neutral elution conditions using ActiSep Elution Reagent (Sterogene, USA). The affinity-purified serum was pre-absorbed against homozygous $y w$; toy ${ }^{\text {hdl }}$ third instar larvae. Adult flies ( $h s$-Gal4>UAS-toy and control) were heat-shocked at $37^{\circ}$ for $45 \mathrm{~min}$, left at room temperature for $30 \mathrm{~min}$, heat-shocked again for $30 \mathrm{~min}$ and protein was prepared after another $45 \mathrm{~min}$ at room temperature. Western blots were done according to standard procedures, using the Toy antiserum as primary antibody (dilution 1:1,000) and HRP-conjugated anti-rabbit antibody (NA934, Amersham) (dilution 1:10,000) as secondary antibody. The immunoreactions were visualized with the ECL+ system (RPN2132, Amersham). Immunohistochemical analyses in embryos were carried out as per the methods of Patel (1994). The Toy antiserum was diluted 1:400 and a biotin-conjugated goat anti-rabbit antibody was used as secondary antibody (dilution 1:300). The mouse anti- $\beta$-gal antibody (Promega) was used at 1:250 dilution, the mouse anti-Elav antibody (Developmental Studies Hybridoma Bank, Iowa) was used at 1:400. Donkey anti-mouse-Cy3 (Jackson Immuno Res. Lab) (dilution 1:1,000) was used as secondary antibody. Larval tissue was checked for Toy protein expression with the anti-Toy antibody (dilution 1:1,000) and donkey antirabbit Cy-3 conjugated antibodies (dilution 1:1,500) (Jackson Immuno Res. Lab).
General procedures and fly stocks

5'-RACE of mRNA, PCR, Northern blots and DNA sequencing were carried out according to standard procedures. In the Northern blot poly(A) ${ }^{+}$RNA was isolated from 2- to 24-h-old wild-type embryos and a ${ }^{32} \mathrm{P}$-labeled $1.73 \mathrm{~kb}$ toy cDNA was used as probe. RNA size markers were from Promega (RiboMark Labeling). Acridine orange staining was carried out according to standard protocols (Wolff 2000). Embryo staining was done as previously described (Patel 1994). Photos were taken using a Zeiss Axiophot microscope equipped with a CCD camera and KAPPA PS $30 \mathrm{C}$ and KAPPA ImageBase software.

For the scanning electron microscopy samples were dehydrated in a series of increasing concentrations of ethanol up to $100 \%$, with at least $20 \mathrm{~min}$ in every step. They were thereafter dried from liquid carbon dioxide by the critical point technique (Polaron E-3000 Critical Point Drying Apparatus, Polaron Equipment Ltd, Watford, UK). The dried specimens were mounted on aluminum stubs with electrically conductive carbon tape (Agar Scientific, Stansted, UK) and coated with gold using a combined system of sputter coating (Edwards S150A Sputter Coating Unit, Edwards High Vacuum Ltd, Crawley, UK) and a modified evaporation coating system with automatic tilting and rotation device (Edwards E14 Vacuum Coating Unit, Edwards High Vacuum Ltd). Microscopy was performed in a scanning electron microscope (LEO 360ixp SEM, LEO Electron Microscopy Group, Oberkochen, Germany).

The following fly stocks were used: Oregon- $R, w^{1118}, w$; toy $^{\text {hdl }} / c^{D}{ }^{D}{ }_{\text {spa }}{ }^{\text {pol }}$ (Kronhamn et al. 2002), y w; toy ${ }^{G 7.39} / c i^{D}$ (Kammermeier et al. 2001), w; ey $y^{D} / i^{D} s p a^{p o l}, y w ; e y^{J 5.71} /$ $c i^{D}{ }^{2 p a^{p o l}}$ (Punzo et al. 2001), w; P\{hs-GAL4\}/CyO, w; $P\{G A L 4-e y . H\} 4-8 / C y O$ (Bonini et al. 1997; Hauck et al. 1999), w; P\{UAS-lacZ\}/TM3, w ${ }^{1118} ;$ P $\{U A S$-GFP.nls $\} 14$, $w ; P\{U A S$-toy $\} / T M 3, w ; P\{U A S$-toy $\} / C y O$ (Czerny et al. 1999), w; P\{UAS-ey.H\} (Halder et al. 1995), w; P\{UASp35.H\}BHI (Hay et al. 1994), w; P\{UAS-RpL14.IR\} (Enerly et al. 2003) and w: P\{GAL4-vg.M\}2; TM3, Ubx/ $T M 6 B, T b$ (Bloomington Drosophila Stock Center).

Rescue crosses were done in vials containing standard medium, at the temperatures indicated $\left(29^{\circ}, 25^{\circ}, 22^{\circ}, 18^{\circ}\right.$ or $\left.15^{\circ}\right)$. Each cross was repeated at least once. We analyzed the rescue of four different Pax6 mutants; $e y^{D}$, toy $y^{h d l}, e y^{J 5.71}$, and toy ${ }^{G 7.39}$. Three different Gal4 driver lines (ey-Gal4 and two toy-Gal4 lines) and five UAS lines (UAS-toy (2 lines), $U A S-e y, U A S-P 35$, and $U A S-G F P$, which served as a negative control) were used. Frequencies of homozygous $\operatorname{Pax}^{-}$ offspring in the $w ; U A S$-Pax $6 ; P a x 6^{-} / c i^{D} s p a^{p o l}$ stocks were also analyzed after development at different temperatures.

Homozygous Pax6 mutant offspring was identified by lack of the $c i^{D}$ wing phenotype. The rescued offspring was repeatedly tested by crosses to wild-type flies at $25^{\circ}$, in 
order to verify the absence of the $c i^{D}$ marker. In no case the marker chromosome was present. Rescued offspring were scored for phenotype of both compound eyes and ocelli. Flies with smaller than normal eyes, ranging from a discernible loss of peripheral facets to only a single remaining ommatidium, were classified as "small eyes". Presence of all three ocelli and at least one of the 6-8 the interocellar bristles was defined as wild-type ocellar phenotype.

\section{Results}

Toy expression during embryonic development

To be able to compare the wild-type expression pattern and that of the transgenic lines carrying presumptive toy regulatory regions, we generated a Toy-specific polyclonal antibody in rabbit. To test the specificity of this antiserum we over-expressed UAS-toy by introducing a heat-shock driven Gal4 transgene and analyzed the fly proteins with Western blot. Four bands appeared in the $w^{1118}$ stock (heat shocked) used as control (Fig. 1a). One of these was extremely strong in the heat-shock treated transgenic flies (Fig. 1a, lane 2). The size of the over-expressed protein was in good agreement with the 59-kDa full-length Toy protein. Homozygous toy ${ }^{\text {hdl }}$ mutant larvae lacked this band (Fig. 1a, lane 3 ). The other bands were, however, still present and were, therefore, considered to be due to unspecific binding of the antiserum. We also performed immuno-histochemical analyses of homozygous toy ${ }^{\text {hdl }}$ mutant embryos (Fig. 1, compare panels $\mathrm{d}$ and e). There was a low level of background staining with the Toy antibody in mutant embryos. The Toy-specific staining in the visual primordia, brain and VNC was, however, missing. Ectopic expression of toy in wing discs using a $v g$-Gal4 driver gave a strong Toy-signal in a vg-specific manner (Fig. 1i), while wild-type discs showed no Toy staining (not shown).

The localization of Toy protein during embryonic and larval development was analyzed using this antiserum. Toy protein was first detected at stage 8 in wild-type embryos (not shown). The initial expression was defined to the posterior procephalic region, i.e., same region as toy transcripts are found with in situ hybridization (Czerny et al. 1999). During embryonic stages 9 and 10, Toy-expressing cells were found in a region covering the ectodermal neuroblasts (Fig. 1b, c). These cells moved dorsally and at stage 13, when neuroblasts invaginate to form the brain, protein staining was seen in the optic lobe primordia and in the larval eye primordium (Bolwig's organ) (Fig. 1d; Supplementary Fig. S1). At stage 15, staining was evident in the primordia of the eye-antennal discs (Fig. 1f). Specific cells in the $\mathrm{VNC}$ were visible already at the beginning of stage 14 and at subsequent stages (Fig. 1g). In eye-antennal discs from wild-type third instar larvae toy expression was visible both posterior and anterior to the morphogenetic furrow and in the medial ocellar region (Fig. 1h). The Toy protein expression pattern is thus not completely in agreement with the in situ hybridization results, where toy mRNA is predominately found anterior to the morphogenetic furrow (Czerny et al. 1999; Kammermeier et al. 2001).

Analyses of the toy regulatory region using Gal4 constructs

In order to identify the cis-regulatory regions of the toy gene, we analyzed the transcription start and the $5^{\prime}$ region of toy. An alternative, but rare, transcription start has been found at 954-bp upstream of the earlier reported toy transcription start (Fig. 2a) (Erich Frei, personal communication). This exon (1') is 162-bp long. We sequenced cDNA from embryos and confirmed that this transcription start is used. However, no EST sequence containing this start has been reported. In over-exposed Northern blots, a rare transcript of $1.8 \mathrm{~kb}$ was detected, which could correspond to this transcript (not shown). Quantification of the bands showed that the transcript represented approximately $4 \%$ of the total toy mRNA in embryos. The cDNA sequence indicated that transcripts initiated at exon $1^{\prime}$ are spliced to exon 2 and translated from an AUG site located in exon 3. Translation of this rare transcript would thus result in a Toy protein that lacks the paired domain.

Six different promoter constructs were generated in order to dissect the $5^{\prime}$ regulation of the toy gene (Fig. 2a). toyl contained 5,203 bp of genomic DNA upstream and $526 \mathrm{bp}$ downstream of the canonical transcription start (exon 1). Constructs toy 2 , toy3, and toy 4 had the same proximal end as toyl but contained 767, 597, and $320 \mathrm{bp}$ of upstream DNA, respectively. Two constructs contained the region upstream of and including the alternative transcription start (exon $\left.1^{\prime}\right)$; toy5 (4,457 bp) and toy6 (1,313 bp).

The regulatory function of the constructs was first analyzed by crossing transformed lines to a stock containing a UAS-RpL14.IR transgene. This is an RNAi construct of the Ribosomal protein L14. By driving this construct with an ey-Gal4 driver (containing the eye-specific enhancer of $e y$ ) Lambertsson and co-workers were able to generate headless flies due to interference with the translational machinery in the head (Enerly et al. 2003). This phenotype is very similar to the headless phenotype of toy ${ }^{\text {hdl }}$ or $e y^{D}$ homozygotes. The results from these crosses are summarized in Supplementary Table S1. The two longest toy-promoter regions containing the exon 1 transcription start (toyl and toy2) resulted in lethality (4 of 11 lines) together with $U A S$ $R p L 14^{R N A i}$ and a headless phenotype of pharate adults (dissected out of the pupal cases) was found in two of these lines. The variation in phenotype between lines of a specific construct indicated position effects at the transgene 

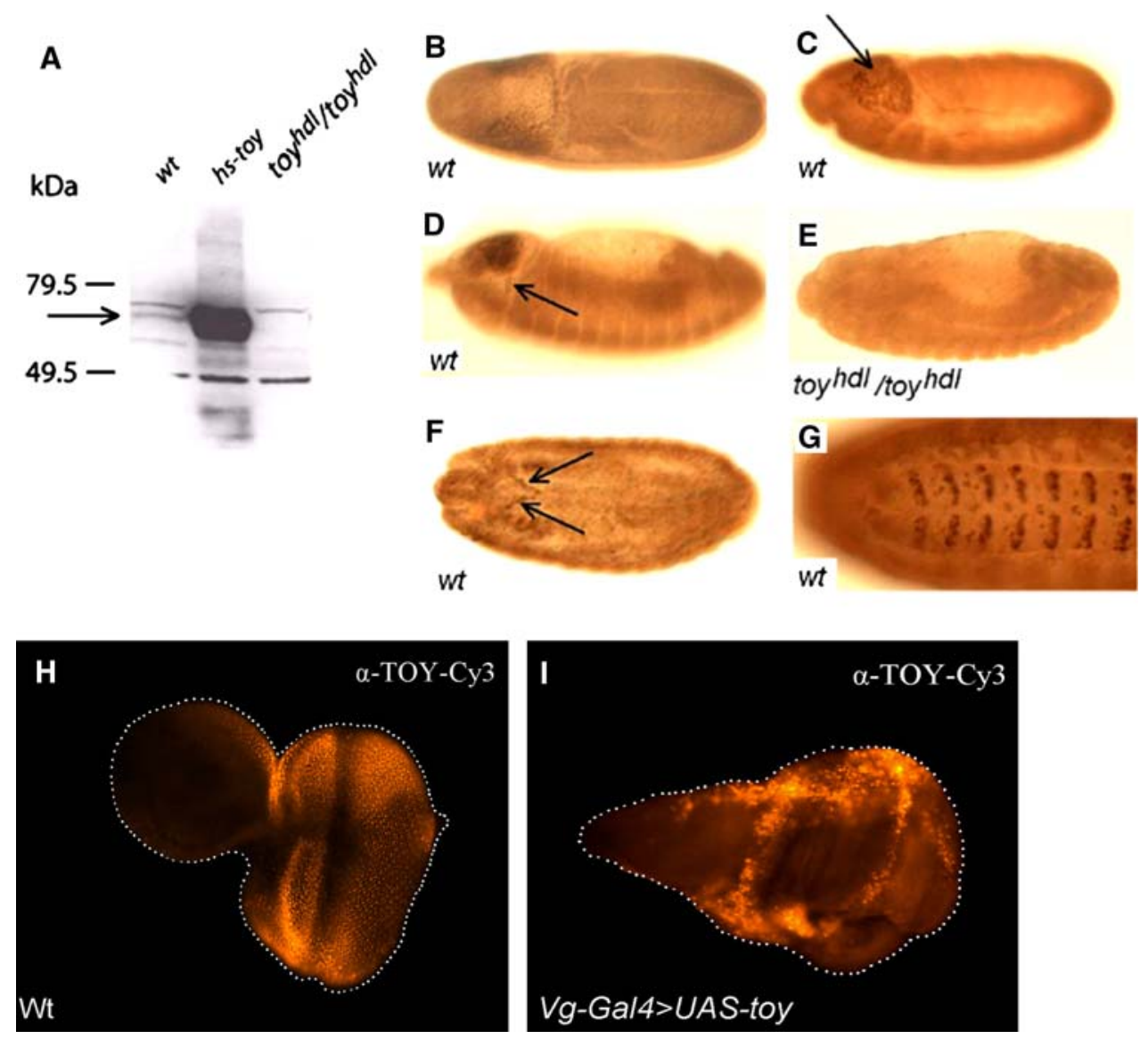

Fig. 1 Toy wild-type expression pattern and loss in the toy ${ }^{\text {hdl }}$ mutant. a Western blot analysis using the Toy antibody. Lane 1 shows proteins prepared from $w^{1118}$ adults, heat-shocked by the same regime as the $h s$ Gal4; UAS-toy adults in lane 2. The Toy protein is visible as a weak band in wild-type, but is strongly over-expressed by heat shock. The arrow indicates the 59-kDa Toy protein. Homozygote toy ${ }^{\text {hll }}$ larvae (lane 3) lack this band, but three weak unrelated bands are still present. b Immunohistochemical staining of Toy in a wild-type embryo (stage 9) showing expression in two circular areas of the head (dorsal view). c Lateral view of a stage 11 wild-type embryo. The Toy-expressing

cells have moved dorsally and form an uneven patch, see arrow. d Lateral view of a late stage 13 wild-type embryo. Arrow shows staining at the Bolwig's organ. e Lack of specific staining is observed in a $y w$; toy $y^{h d l}$ embryo (stage 14). Compare with the wild-type expression pattern in d. f Dorsal view of a stage 15 wild-type embryo. Arrows point at the V-shaped eye-antennal primordia. $g$ The reiterated pattern of Toy in the ventral cord of a stage 16 wild-type embryo. h Toy expression pattern in a wild-type eye-antennal disc. i Ectopic expression of Toy in a wing disc using the $v g$-Gal4 driver

insertion sites, a notion strengthened by the variable expression of the white ${ }^{+}$reporter gene, which was variegated or very weak in most lines viable with $U A S-R p L 14^{R N A i}$. None of the other tested constructs gave lethality with $U A S$ $R p L 14^{R N A i}$, indicating that a DNA region of at least $1.3 \mathrm{~kb}$ surrounding the canonical transcription start (toy2) is important for correct Toy expression during head development.

We also crossed a portion of the transgenic toy-Gal4 lines to reporter constructs (UAS-lacZ and UAS-GFP) to analyze the embryonic, larval, and adult expression patterns. The results are summarized in Supplementary Table S1. The embryonic expression was analyzed with lac $Z$ staining using an anti- $\beta$ gal antibody. Many of the toy 1 and toy 2 lines showed specific expression of lac $Z$ in the brain (Fig. 2b-e) and in the eye-antennal primordia (Fig. 2c, only one primordium is in focus) resembling the

normal expression of toy. One of five toy3 lines also showed this expression pattern. The shortest construct (toy4) and the two constructs using the upstream transcription start did not give this pattern, indicating that an embryonic eye-antennal specific enhancer is located within the 1,123-bp-region surrounding the canonical transcription start site. VNC staining was found in toyl, toy2, and toy3 lines, but not with the other transgenic constructs. We also found unexpected $L a c Z$ stainings in some lines, e.g., expression of all the toy 5 and toy 6 Gal 4 driver constructs was confined to one pair of cells in the ventral part of the head during early embryogenesis. Other lines stained cells in the amnioserrosa and cells reminiscent of embryonic hemocytes. The latter patterns were not consistent between lines of a specific construct and were therefore considered as caused by position effects. 
Fig. 2 Analysis of the 5-kb region upstream of toy using reporter constructs. a Map of the upstream and promoter region of toy showing the Gal4 constructs toy 1 to 6 . A newly identified transcription start site is marked $1^{\prime}$. The $5^{\prime}$ untranslated region is indicated with white boxes and the first part of the toy open reading frame is black. The striped box is translated when spliced to exon 1, but not if transcription starts at exon $1^{\prime}$. Below the map, extents of genomic sequences inserted into Gal4 vectors and used for transformation of flies are shown. b Reporter gene expression in a stage 10 embryo of toyla-Gal4>UAS$L a c Z$ showing neuroblast expression. c A stage 16 embryo of the same strain showing staining in the embryonic brain and in one eye-antennal primordium (arrow). d A toyle-Gal4>UASLacZ embryo (stage 10) showing an early expression of the lac $Z$ reporter gene. e A toyla-

Gal4>UAS-EGFP eye-antennal disc showing strong expression of the GFP reporter gene in a band anterior to the morphogenetic furrow and in the ocellar region and weak expression in a few spots in the antennal portion
A

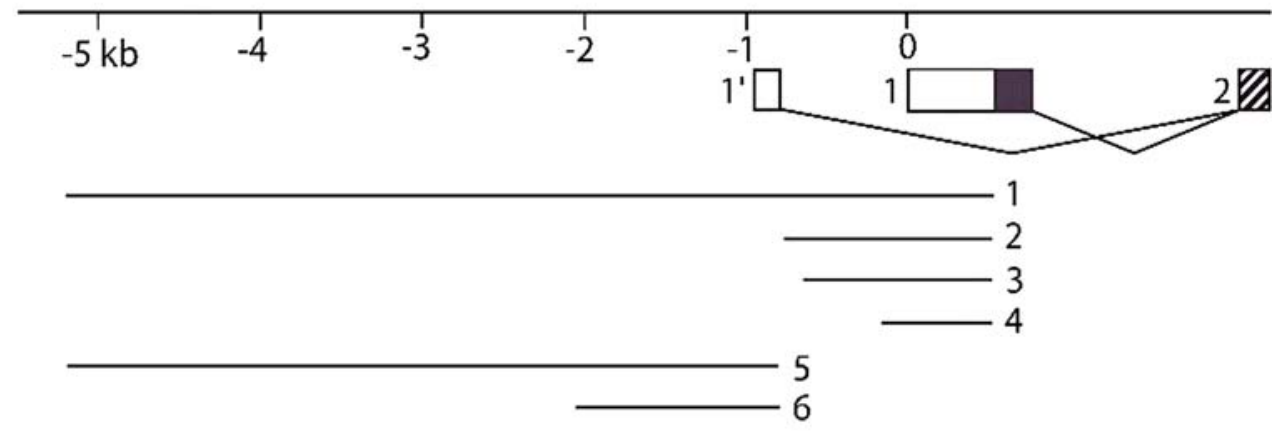

B

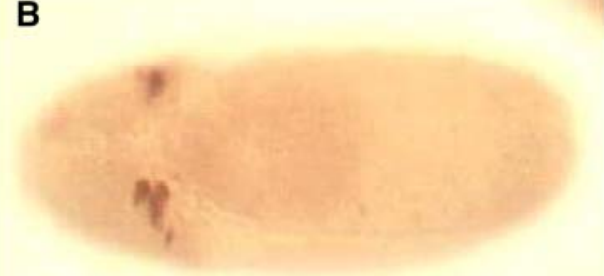

toy1a-Gal4> UAS-lacZ

D

toy $1 e-G a 14>$ UAS-lacZ
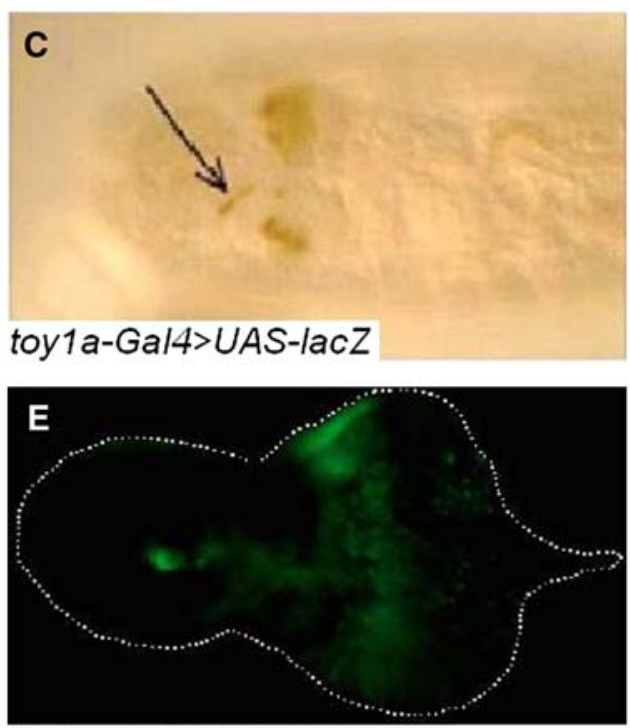

toy1a-Gal4>UAS-EGFP
We also analyzed larval expression of the toy regulatory constructs in the eye-antennal imaginal discs and brains. These results are summarized in Supplementary Table S1. The regulatory regions present in toy 1, toy 2 , toy 3 , and toy 4 constructs induced GFP expression in eye-antennal discs (5 of 17 lines) and/or in brain (5 of 17 lines). The discs of toyl-Gal4 (Fig. 2e) and toy2-Gal4 show GFP expression anterior to the morphogenetic furrow, resembling the Toy wild-type expression pattern, while toy 3 and toy 4 gave signals only at the posterior edge of the eye disc (Supplementary Fig. S2). The other tested transgenic lines showed no fluorescence in either discs or brain. Furthermore, exterior examination of GFP expression revealed fluorescence in brains of adult flies carrying toy 1 or toy 2 constructs ( 4 of 5 lines tested).

Alignment studies of the toy upstream region between D. melanogaster and D. pseudoobscura did not reveal much conservation. However, using the human Pax6 consensus binding site in ConSite (Lenhard et al. 2003) we identified two putative Pax6-binding sites flanking the canonical transcription start site. These sites were conserved in seven closely related Drosophila species within the melanogaster and obscura groups, but they were not present in the more distantly related willistoni, virilis, and repleta groups. Neither were they present at the transcription start site or in the coding sequence of the eyeless gene. We also noted a presumptive Bicoid binding site $250 \mathrm{bp}$ upstream of the toy transcription start.

In summary, the results indicate that a $1.3-\mathrm{kb}$ region surrounding the transcription start site is important for correct Toy expression during embryonic and larval development. However, transcription from the alternative transcription start site (exon $1^{\prime}$ ), does not seem to play a major role for the expression of Toy and was not studied further. With the use of toyl and toy 2 Gal4 driver constructs we set out to induce targeted expression of toy and ey cDNAs in order to rescue some strong Pax6 mutants (toy ${ }^{\text {hdl }}$, toy ${ }^{G 7.39}$, ey $y^{J 5.71}$ and $e y^{D}$ ).

\section{Rescue of toy mutants}

As shown earlier, about one-third of the homozygous toy ${ }^{h d l}$ mutants die during embryonal or larval stages and the remaining two-thirds reach the pupal stage, where many 
develop as far as pharate adults (Kronhamn et al. 2002). At this stage they die since they are unable to escape from the pupal case. Almost no escapers eclose at $25^{\circ}$ or at higher temperatures. If balance stocks are allowed to grow at $18^{\circ}$, about $3 \%$ of the eclosing flies are homozygous mutant with normal head morphology, apart from the ocelli, which are aberrant or missing (Kronhamn et al. 2002). Removal of the pupal cases shows flies with variable head defects, ranging from almost normal heads with small compound eyes, over "half-heads" and "cleft-heads" (Fig. 4f), to completely headless flies (Fig. 4h) where the proboscis is protruding from the thorax. The toy ${ }^{h d l}$ mutant is caused by a deletion uncovering exons 6-9 of toy. This would theoretically result in a protein with a truncated homeodomain but with an intact paired domain. Antibody staining, however, showed no protein in homozygote toy ${ }^{\text {hdl }}$ embryos or larvae (Fig. 1). For the rescue experiments we used the toy ${ }^{\text {hdl }}$ allele and a hypomorphic allele; toy ${ }^{G 7.39}$ (Punzo et al. 2002), which also exhibits a headless phenotype very similar to toy ${ }^{\text {hdl }}$ homozygotes. Staining with the Toy antibody showed that Toy protein is below detection level in the eye-antennal primordia of homozygous toy ${ }^{G 7.39}$ embryos; however, we found expression in the VNC (not shown), indicating that the toy ${ }^{G 7.39}$ mutant is an eye-specific allele.

To optimize the temperature sensitivity property of the Gal4/UAS system (Jarrett 2000) flies are normally raised at $29^{\circ}$ (Brand and Perrimon 1993), which provides a balance between maximal Gal4 activity and minimal detrimental effects on fertility and viability. However, at this temperature the effect of driver and/or responder was so strong that no flies survived (Fig. 3). By decreasing the developmental temperature to $25^{\circ}$, we found an eclosion rate of rescued homozygous toy ${ }^{\text {hdl }}$ flies of about $3.5 \%$ (Fig. 3, black bar) compared to $4 \%$ in flies without rescuing transgenes (light gray bar) and $0.9 \%$ in balanced stocks (white bar). The survival was, thus, not significantly different with or without rescuing constructs. Neither did we find any significant rescue at $22^{\circ}$. At $18^{\circ}$ and $15^{\circ}$, however, the frequency of eclosing homozygotes increased significantly to about $20-23 \%$ in flies carrying both toy-Gal4 (toyla or toy $2 b$ ) and UAS-toy constructs (Fig. 3, black bars). The expected frequency of toy ${ }^{\text {hdl }} /$ toy ${ }^{\text {hdl }}$ adults at full rescue was $33 \%$. Unexpectedly, we found an equally high rescue level of homozygouos toy ${ }^{\text {hdl }}$ flies carrying the UAS-toy construct alone (dark gray bars). The experiments were repeated with five different toy-Gal4 lines and two different UAS-toy lines, and in all cases the rescue of toy ${ }^{\text {hdl }}$ homozygotes was comparable, irrespective of whether the toy-Gal4 driver was present or not. The rescue at these lower temperatures was, however, significantly higher than the number of homozygotes without UAS-toy constructs (Fig. 3, white, light gray and medium gray bars). The results also indicated that there was a positive effect on survival obtained

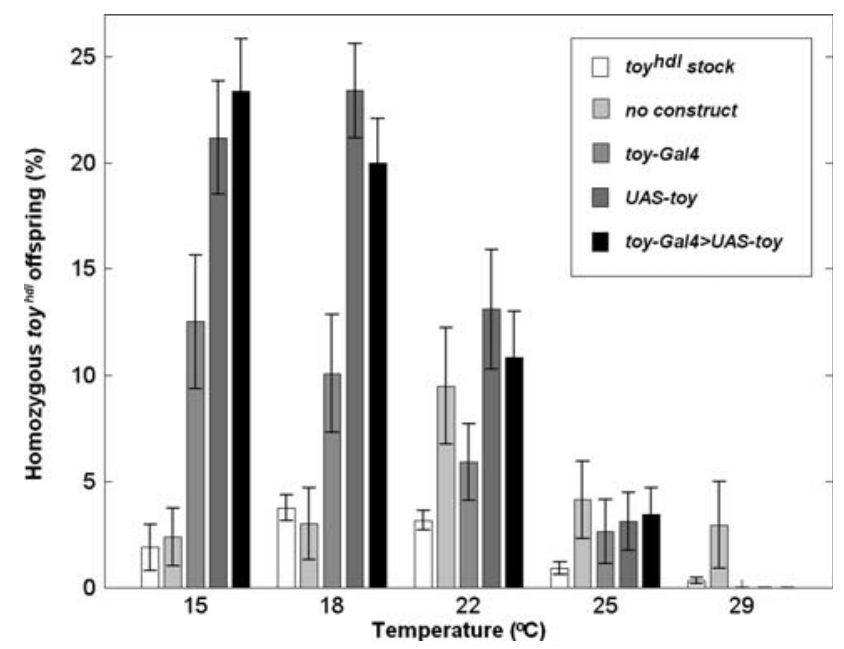

Fig. 3 Rescue from lethality of toy hdl homozygotes with toyGal4>UAS-toy at different developmental temperatures. White bars toy ${ }^{\text {hdl }} / \mathrm{ci}^{D} \mathrm{spa}^{\text {pol }}$ stock (control). Genotypes after crossing: light gray no rescuing constructs (control); medium gray toy-Gal4; dark gray UAStoy; black toy-Gal4>UAS-toy. Significant rescue compared to controls $\left(\chi^{2}\right.$ test; $\left.P<0.001\right)$ was obtained at $15^{\circ}$ and $18^{\circ}$ with toy-Gal4>UAStoy but also with UAS-toy alone. The figures are based on the total of toy $1 a$ and toy $2 b$ rescue cross results since these figures did not differ significantly. Error bars show SD

by just crossing flies, since the classes of offspring carrying no construct or only toy-Gal4 also showed a weak increase in survival compared to balanced stocks at some temperatures. This effect was, however, not found when we examined survival of toy hdl homozygotes carrying toylaGal4>UAS-GFP. The number of viable adult offspring in these crosses was not significantly different from the level of escapers in the $w$; toy $^{h d l} / c i^{D} s p a^{p o l}$ stock (not shown), indicating that toy-Gal4 did not cause rescue by itself. Using the same construct (toyla-Gal4>UAS-toy) we were also able to rescue homozygous toy ${ }^{G 7.39}$ mutant flies with similar efficiencies as the toy ${ }^{\text {hdl }}$ mutant (Table 1 and not shown). To explore whether the UAS-toy transgene was transcribed also without a Gal4 driver construct, we made quantitative RT-PCR analyses of flies carrying the UAS-toy with and without driver in a toy ${ }^{\text {hll }}$ background (Supplementary Fig. S3). The flies reared at $25^{\circ}$ showed increased levels of toy transcripts whenever UAS-toy was present (also without a toy-Gal4 driver construct). In these flies we also observed a weak increase in ey transcription. The results indicate that the UAS-toy construct used was leaking.

Apart from the increase in survival of homozygous toy mutant flies, we also found an improvement of the ocellar phenotypes of the rescued flies when combining toy-Gal4 with UAS-toy (Table 1; Fig. 4a, b). A similar rescue of the ocelli formation was found in flies carrying the UAS-toy construct alone, while flies only carrying the driver still lacked ocelli. The proportion of flies with normal ocelli among the homozygous escapers in a toy ${ }^{h d l} / c i^{D} s p a^{p o l}$ stock 
Table 1 Rescue of lethality and eye phenotypes in Pax6 mutants with exogenous Pax6 (18 $)$

\begin{tabular}{|c|c|c|c|c|c|c|c|}
\hline \multirow[t]{2}{*}{ Mutant } & \multirow[t]{2}{*}{ Rescuing construct ${ }^{\mathrm{a}}$} & \multirow{2}{*}{$\begin{array}{l}\text { Fraction of } \\
\text { homozygote } \\
\text { adults }(\%)^{\mathrm{b}}\end{array}$} & \multirow[t]{2}{*}{$\begin{array}{l}\text { Total no. of } \\
\text { offspring scored }\end{array}$} & \multicolumn{3}{|c|}{$\begin{array}{l}\text { Homozygote compound } \\
\text { eye phenotype }(\%)^{\mathrm{c}}\end{array}$} & \multirow[t]{2}{*}{$\begin{array}{l}\text { Homozygotes }(\%) \\
\text { with normal ocelli }^{\mathrm{d}}\end{array}$} \\
\hline & & & & + & \pm & - & \\
\hline \multirow[t]{3}{*}{ toy $y^{h d l}$} & - & 3.75 & 1,013 & 100 & 0 & 0 & 18 \\
\hline & toy-Gal4>UAS-toy & $14.75 * * *$ & 746 & 100 & 0 & 0 & 50 \\
\hline & toy-Gal4>UAS-ey & $14.08 * * *$ & 419 & 100 & 0 & 0 & 83 \\
\hline \multirow[t]{3}{*}{ toy $y^{G 7.39}$} & - & 5.10 & 471 & 100 & 0 & 0 & 71 \\
\hline & toy-Gal4>UAS-toy & $20.69 * * *$ & 667 & 100 & 0 & 0 & 90 \\
\hline & toy-Gal4>UAS-ey & $9.53 * *$ & 599 & 100 & 0 & 0 & 85 \\
\hline \multirow[t]{3}{*}{$e y^{D}$} & - & 0.27 & 366 & 0 & 0 & 100 & 100 \\
\hline & toy-Gal4>UAS-toy & $7.30 * * *$ & 233 & 0 & 76 & 24 & 100 \\
\hline & ey-Gal4>UAS-ey & $3.01 * *$ & 432 & 0 & 77 & 23 & 100 \\
\hline \multirow[t]{2}{*}{$e y^{J 5.71}$} & - & 12 & 175 & 0 & 90 & 10 & 95 \\
\hline & toy-Gal4>UAS-toy & $26.8 * * *$ & 239 & 100 & 0 & 0 & 81 \\
\hline
\end{tabular}

a Toy-Gal4 drivers could be either line toyl $a$ or toy $2 b$

${ }^{\mathrm{b}}$ Observed fractions of flies homozygous for the Pax6 mutation indicated to the left (i.e., lacking the $c i^{D} s p a^{p o l}$ chromosome). The expected fraction of homozygous adults at full rescue was $33 \%$. Statistical comparison was made with $\chi^{2}$ test, comparing with the frequency of escapers without rescuing construct

${ }^{c}+$ wild-type compound eyes (examples in Fig. 4a, b), \pm smaller than normal eyes (example in Fig. 4c) (similar to $e y^{2}$ ), - no compound eye present (example in Fig. 4e)

d Three normally positioned ocelli present (examples in Fig. 4a, b, e)

*** $P<0.001, * * P<0.01$

was $18 \%$ at $18^{\circ}$. However, in rescued flies, this proportion increased to $50 \%$. The ocellar phenotype of toy ${ }^{G 7.39}$ mutant homozygotes was weaker ( $71 \%$ normal flies) than that of toy ${ }^{\text {hdl }}$, but a phenotypic improvement was achieved with an exogenous expression of UAS-toy (Table 1).

To conclude, we obtained a significant amelioration of the survival and the head phenotype of homozygous toy mutant flies with a transgenic copy of UAS-toy at lower temperatures $\left(15^{\circ}\right.$ and $\left.18^{\circ}\right)$ while at higher temperatures survival was not improved. We find that the UAS-toy transgenic construct can induce transcription of its own at higher temperatures. Thus, the presence of the toy-Gal4 driver construct is not necessary to obtain rescue of the toy hdl mutant. However, at higher temperatures the Toy levels probably are too high to promote development.

Recue of $e y^{D}$

It has been shown that full-length ey cDNA is able to efficiently rescue the eye phenotype in $e y^{2}$ mutants when expressed in the eye disc under the control of the eye-specific ey enhancer (Halder et al. 1998). Punzo et al. (2002) also reported a rescue of the $e y^{J 5.71}$ allele using eyGal4>UAS-ey. The $e y^{J 5.71}$ allele is caused by a $9-\mathrm{kb}$ deletion in the $5^{\prime}$ region of the gene and is described as an RNA and protein null mutant (Punzo et al. 2001). Both $e y^{J 5.71}$ and $e y^{D}$ mutations cause homozygous lethality (ey $y^{J 5.71}$ to $81 \%$ ); however, in contrast to $e y^{D}$, homozygous $e y^{J 5.71}$ adults are not headless. First, we wanted to test whether it was possible to rescue homozygous $e y^{D}$ pharates by targeted expressing of Ey. Using a driver construct consisting of the eye-specific enhancer of the ey gene (Bonini et al. 1997; Halder et al. 1998) combined with an UAS-ey transgene, we were able to significantly rescue $e y^{D}$ homozygotes (Table 1 ). The flies that eclosed from pupae exhibited smaller than normal heads and a majority of the compound eyes were rescued to a size comparable to that of $e y^{2}$ mutants (Fig. 4c) while ocelli were normal (Table 1). As observed in the toy ${ }^{\text {hdl }}$ rescue, we found that viability rescue of $e y^{D}$ was more efficient at $18^{\circ}$ compared to $25^{\circ}$ and $29^{\circ}$ and also that rescue efficiencies of $e y^{D}$ flies carrying both driver and effector constructs (ey-Gal4>UAS-ey) or effector construct alone (UAS-ey) were similar (not shown). This was found for both survival and compound eye phenotype.

To conclude, the results show that exogenous expression of Ey can partially rescue both lethality and compound eye structure of the dominant-negative mutant $e y^{D}$, in analogy with previous results for other ey mutants (Punzo et al. 2002).

Toy and Ey can substitute for each other but have distinct functions in head formation

Targeted expression of Ey can induce ectopic eye formation, without induction of toy (Czerny et al. 1999). Moreover, it has been shown that Toy can activate eye 

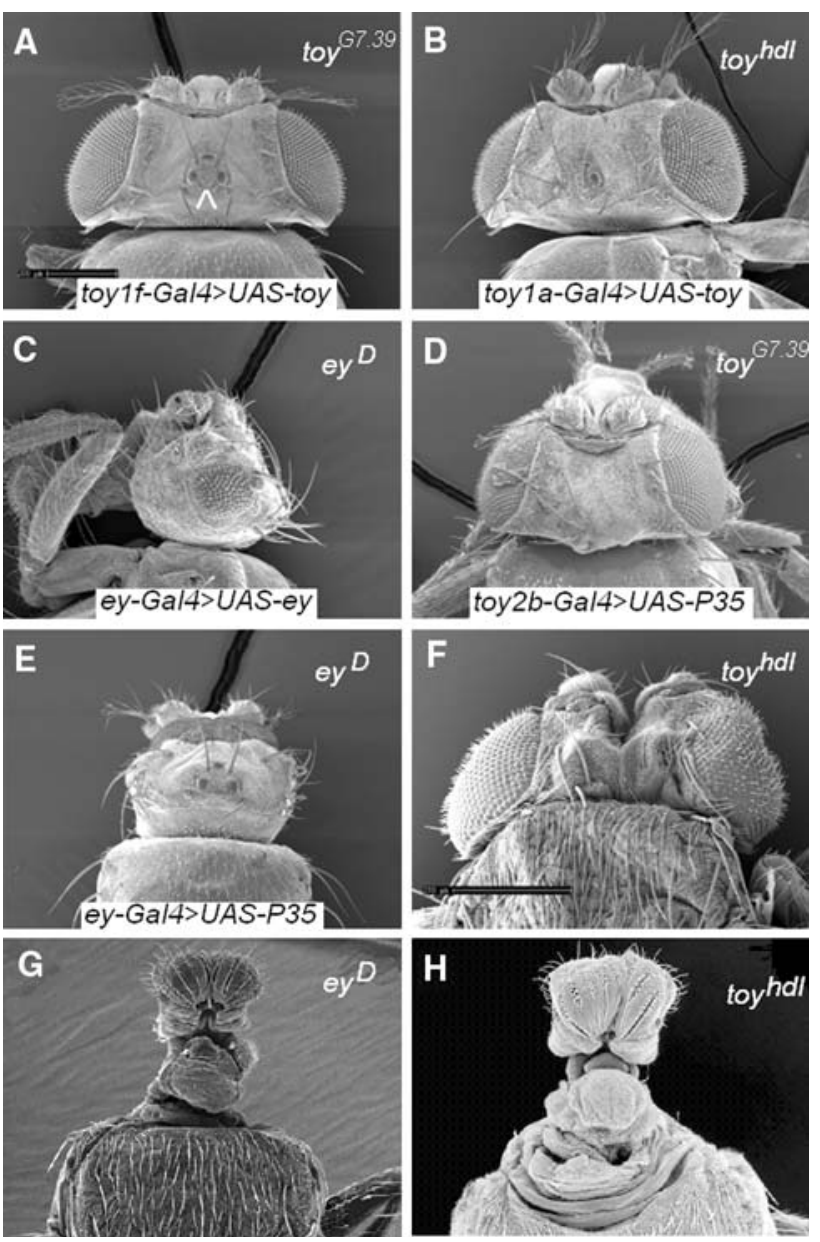

Fig. 4 Scanning electron micrographs of heads from toy and ey mutants and rescued flies. a Rescue of homozygous toy ${ }^{G 7.39}$ with toylfGal4>UAS-toy. The phenotype is indistinguishable from wild type. The interocellar bristles are marked with an arrowhead. b Rescue of toy ${ }^{\text {hdl }}$ with toyla-Gal4>UAS-toy. The ocellar phenotype is classified as wild type since it has three ocelli and a few interocellar bristles in the correct positions. c Rescue of $e y^{D}$ with $e y-G a l 4>U A S$-ey. d Rescue of homozygous toy ${ }^{G 7.39}$ with the anti-apoptotic protein P35 (toy $2 b$ Gal4>UAS-P35). Compound eyes are wild type, but ocelli are missing. e Rescue of $e y^{D}$ with P35 (ey-Gal4>UAS-P35). Compound eyes are missing, but ocelli are normal. a-e All flies have all eclosed from the pupal case. f A homozygous toy ${ }^{\text {hdl }}$ pharate adult showing a weaker head phenotype "cleft head". g Homozygous headless $e y^{D}$ pharate adult. h Homozygous headless toy ${ }^{h d l}$ pharate adult. $\mathbf{f}-\mathbf{h}$ Pharate adults dissected out of the pupal cases. $\mathbf{a}-\mathbf{e}, \mathbf{g}$ are the same scale. Bars $200 \mu \mathrm{m}$

development in an ey-independent manner (Punzo et al. 2004). Both toy and ey are expressed in the embryonic eye primordium and later on in the eye-antennal discs. Furthermore, both proteins regulate the eye-specific enhancer of sine oculis, by binding to partly overlapping binding sites (Punzo et al. 2002). In spite of the fact that there are differences in the expression patterns in brain, VNC and in the ocellar territory of the eye discs, these findings indicate that the two Pax6 genes act in parallel and have redundant functions in eye development. To investigate such a redundancy, we set up crosses to rescue homozygous toy and ey mutants, respectively, through ectopic expression of the paralogous Pax6 protein.

We found that the rescue from pupal lethality of the toy ${ }^{\text {hdl }}$ mutant by expression of UAS-ey was as effective as rescue with UAS-toy (14\%) when using the toyla-Gal4 driver (Table 1). Also, the survival of toy ${ }^{G 7.39}$ homozygotes was improved by ectopic expression of UAS-ey. For both toy mutants, the ocellar phenotype was partially rescued. Furthermore, the rescue of $e y^{D}$ homozygotes was even more efficient with toyla-Gal4>UAS-toy (7\%) than with eyGal4>UAS-ey over-expression (3\%) (Table 1). Ectopic expression of toy in the ey ${ }^{J 5.71}$ mutant also gave a significantly increased survival at $18^{\circ}$ (Table 1 ). Compound eyes of $e y^{J 5.71}$ homozygotes were fully restored by one copy of UAS-toy (Table 1). The morphology of compound eyes in rescued $e y^{D}$ mutants was also improved by targeted expression of toy, but never to wild-type proportions. A small eye phenotype was found in $76 \%$ of rescued $e y^{D}$ flies, while without rescue the rare surviving homozygous flies never develop compound eyes. All these experiments were also performed at $22^{\circ}$ with similar results (not shown).

To verify that increased toy levels did not inadvertently induce transcription from the $e y^{J 5.71}$ gene, we made RTPRC analyses. We found a significant increase of ey mRNA levels in UAS-toy; toy ${ }^{\text {hdl }}$ flies, but no increase in UAS-toy; $e y^{J 5.71}$ flies (Supplementary Fig. S3). We conclude that $e y^{J 5.71}$ is not induced by over-expression of toy, indicating that Toy protein can promote head and eye development in an Ey-independent manner, as proposed earlier (Punzo et al. 2004).

In summary, Pax6 proteins can substitute for each other concerning both head structures and survival. However, rescue is only partial; indicating either that the proteins are not completely interchangeable or that the levels of expression and the spatial and temporal requirements are not adequately reproduced by the artificial Gal4-UAS system.

Cell death in imaginal discs of toy mutants and rescue with P35

Both $e y^{D}$ and toy hdl homozyogous mutants have a headless phenotype (Kronhamn et al. 2002) which results in pupal lethality. The eye phenotypes of the escaping or rescued flies are, however, very different, with toy mutants exhibiting defective ocelli while ey mutants lack or have small compound eyes. This indicates that neither Pax6 gene can substitute for the other completely but display a certain degree of subfunctionality. To further corroborate this difference, we analyzed morphology of eye-antennal discs in $t o y^{h d l}$ and $e y^{D}$ mutant larvae and stained the discs with acridine orange to examine levels and localization of cell death 
Fig. 5 Toy expression and cell death in eye-antennal discs. a Acridine orange $(A O)$ staining of a wild-type eye-antennal disc. Arrow points at the morphogenetic furrow in the eye disc. b Eye-antennal disc from a homozygous $e y^{D}$ mutant larva showing a strongly reduced eye portion (right part) with high levels of cell death. c, $\mathbf{d}$ Eyeantennal discs from two homozygous toy ${ }^{\text {hdl }}$ mutant larvae with almost wild-type phenotype except an increased level of cell death in the posterior part of the antennal portion. e A homozygous toy ${ }^{\text {hdl }}$ disc showing complete loss of the antennal portion and increased levels of cell death in the anterior part of the eye portion. $\mathbf{f} \alpha$-Elav staining of a pair of eye-antennal discs from a homozygous toy ${ }^{\text {hdl }}$ mutant larva showing asymmetric loss of one antennal portion and size reduction of the eye portion. All discs are third instar larval eye-antennal discs with anterior to the left
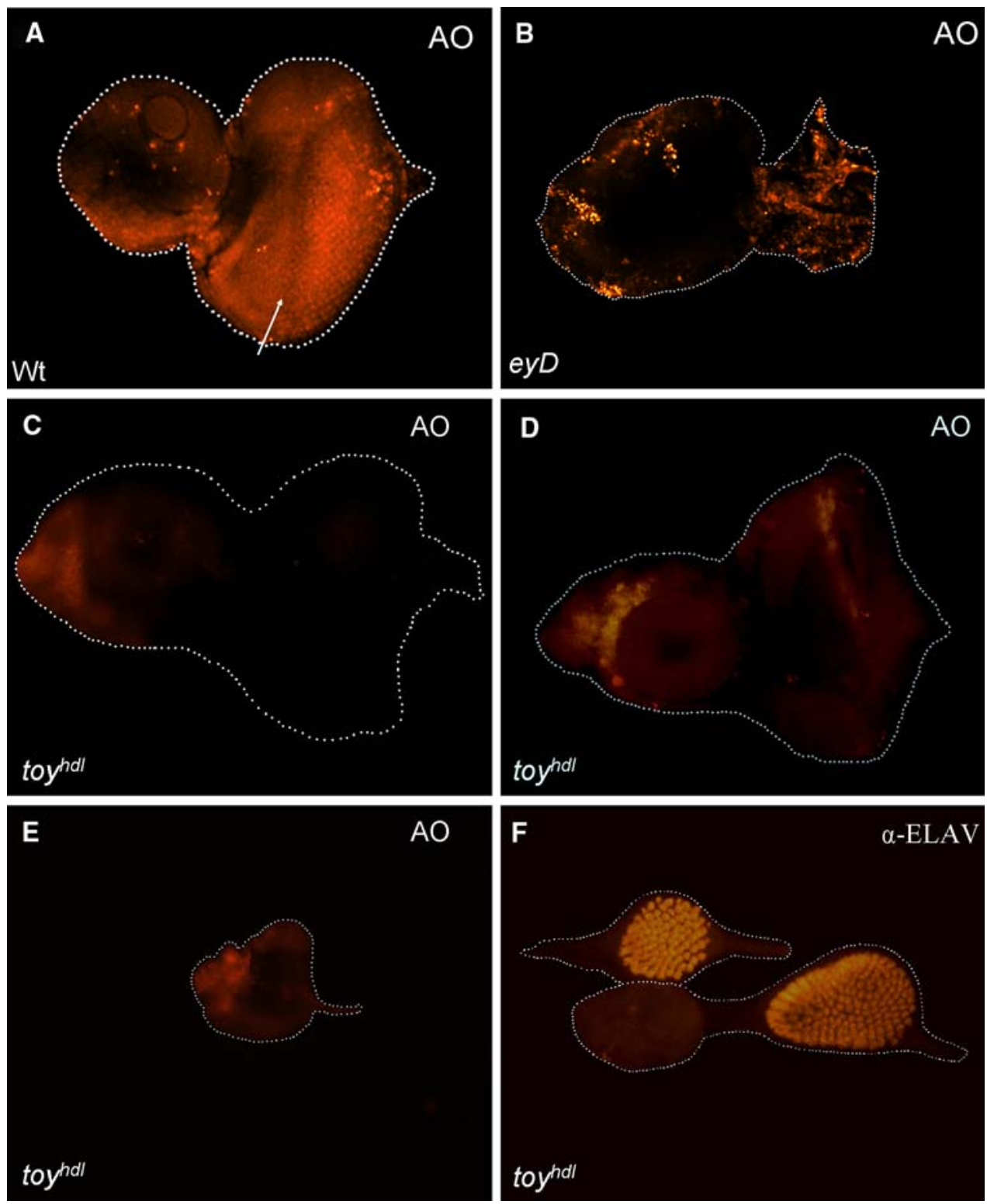

When dissecting eye-antennal discs from toy ${ }^{h d l}$ homozygous larvae grown at $21^{\circ}$, the variability of disc morphology was striking. Fully normal eye-antennal discs were found, as well as defects ranging from size reduction, deformation (Fig. 5c, d), lack of parts (Fig. 5e, f) to complete loss of discs. Asymmetric loss of disc portions was often seen. To verify which part of the discs was missing, we used the neuronal marker $\alpha$-Elav, which stains the differentiated neurons in the eye portion of the disc. In Fig. 5f, a pair of eye-antennal discs from a homozygous toy ${ }^{\text {hdl }}$ third instar larva is shown where the antennal part of one disc is completely missing and the eye portion is smaller. Duplications of the antennal disc were also observed. The frequency of defects was in good agreement with the phenotypes of pharate adults found at this temperature (Kronhamn et al. 2002).
Staining of wild-type eye-antennal discs with acridine orange revealed a low level of cell death in the eye part as expected (Fig. 5a). In the antennal portion, these larvae showed small discrete regions of acridine orange staining. In homozygous toy ${ }^{\text {hdl }}$ larvae staining was relatively normal in the eye portion of most discs at $21^{\circ}$. In the antennal part of the discs, on the other hand, massive cell death was often observed (Fig. 5c, d). In cases where the antennal part was gone, cell death seemed to spread into the anterior edge of the eye portion reaching the region where the frons and ocelli precursor cells are localized (Fig. 5e). We also stained discs from $e y^{D}$ homozygous larvae and found a massive cell death which was most prominent in the remaining portion of the eye discs (Fig. 5b). This is comparable to the acridine orange staining reported in $e y^{2}$ mutants (Halder et al. 1998). 
Table 2 Rescue of lethality of Pax6 mutants with exogenous P35 $\left(18^{\circ}\right)$

\begin{tabular}{llll}
\hline Mutant & Rescuing construct & $\begin{array}{l}\text { Fraction of } \\
\text { homozygote } \\
\text { adults }(\%)^{\mathrm{a}}\end{array}$ & $\begin{array}{l}\text { Total no } \\
\text { of offspring } \\
\text { scored }\end{array}$ \\
\hline toy $^{\text {hdl }}$ & - & 3.75 & 1013 \\
& UAS-P35 & 6.57 & 137 \\
& toy2b-Gal4; UAS-P35 & $19.6^{* * *}$ & 133 \\
toy $^{G 7.39}$ & - & 5.10 & 471 \\
& UAS-P35 & 8.48 & 672 \\
\multirow{4}{*}{ ey $^{D}$} & toy2b-Gal4; UAS-P35 & $16.6^{* * *}$ & 295 \\
& - & 0.27 & 366 \\
& UAS-P35 & 0 & 42 \\
& ey-Gal4; UAS-P35 & $15.6^{* * *}$ & 122 \\
\hline
\end{tabular}

a Observed fraction of viable flies homozygous for the Pax6 mutation (i.e., lacking the $c i^{D} \mathrm{spa}^{\mathrm{pol}}$ chromosome). The expected fraction of homozygous adults at full rescue was $33 \%$. Statistical analyses were made with $\chi^{2}$-test, comparing with the frequency of escapers without rescuing construct

$* * * P<0.001$

We have previously shown that targeted expression of the baculovirus P35 protein, an inhibitor of apoptosis (Hay et al. 1994; Wolff 2000), could rescue the pupal lethality of $e y^{D}$ homozygotes (Kronhamn et al. 2002). Since we found a considerable amount of cell death in the eye-antennal imaginal discs in homozygous toy ${ }^{\text {hdl }}$ larvae, we investigated whether apoptosis inhibition could rescue also the toy mutant phenotypes. We expressed P35 protein under the shorter toy promoter, toy $2 b-G a l 4>U A S-P 35$, in homozygous toy ${ }^{\text {hdl }}$ and toy ${ }^{G 7.39}$ flies, respectively, and found that it was possible to significantly increase survival of both mutants (Table 2 and not shown). In contrast to the results with UAS-toy, however, we found that the ocellar defects of the rescued flies did not improve (Fig. 4d). Another difference was that both driver (toy-Gal4) and effector (UAS$P 35$ ) had to be present to increase survival (Table 2). For comparison, we repeated the P35-induced rescue of $e y^{D}$ and obtained an increase in viability (Table 2 ) and improved head structures (except compound eyes) (Fig. 4, compare e and g) as previously reported (Kronhamn et al. 2002).

These results indicate that the pupal lethality of both toy and $e y^{D}$ mutants is caused by cell loss due to apoptosis, and that inhibition of cell death can improve head structures sufficiently for the pharate adults to escape from the pupal shell. The visual system phenotypes are, however, not rescued by inhibition of apoptosis.

\section{Discussion}

To date, seven genes (toy, ey, eya, eyg, dac, optix, and tsh) (Halder et al. 1995; Czerny et al. 1999; Bonini et al. 1997:
Jang et al. 2003; Shen and Mardon 1997; Seimiya and Gehring 2000; Pan and Rubin 1998) have been considered to be important factors for eye specification in Drosophila. All of them could be called "master control gene of eye development" since it is possible to induce ectopic eyes by misexpression of any of them. Genetic and molecular studies show that these proteins do not lie in a linear biochemical pathway, but rather form an elaborate regulatory network. Our results strengthen this picture since we find that Ey can partially substitute for Toy and vice versa.

\section{Alternative toy transcription start}

Pax6 genes encode nuclear transcription factors, which are characterized by two structurally conserved DNAbinding domains: the paired domain and the paired-like homeodomain. Vertebrates often possess multiple protein isoforms derived from a single Pax6 gene, which can result in distinct expression in different tissues. In mammals, the Pax6(5a) isoform, containing a variant Paired domain with altered DNA recognition (Epstein et al. 1994) has been detected predominantly in eye tissue, while other isoforms are expressed more generally (Jaworski et al. 1997). In C. elegans, an isoform of Pax6, without the paired domain, was found to be important for correct development of the peripheral nervous system (Zhang and Emmons 1995). Punzo et al. (2001) reported that the homeodomain of the Ey protein is dispensable for eye development in flies, but instead have a repressive property directed against the Distal-less protein. The discovery of a new transcription start site in the toy gene in Drosophila, which could be translated into a protein lacking the paired domain, is therefore of interest. The analyses of its expression using the toy5- and toy6-Gal4 driver constructs highlighted a single pair of cells, which seemed to be in contact with the VNC in the posterior part of the embryonic head and could possibly cluster with the labial sensory complex important for formation of the peripheral nervous system of the head. This transcript is, however, not extensively expressed and we are reluctant to ascribe this alternative transcription start site any vital importance in eye or CNS formation.

\section{The 5-kb cis-regulatory region of toy}

Analyses of the pheno-critical periods during development for eye induction implicate that there are two different signals initiating toy transcription: one occurring during the blastoderm stage (Kronhamn et al. 2002) and the other during the second larval instar (Kumar and Moses 2001; Kenyon et al. 2003). Our aim was to study the expression patterns of the $5-\mathrm{kb}$ cis-regulatory region of the toy gene. In addition, we analyzed this sequence for presumptive 
protein-binding sites in order to understand how the expression of toy is regulated.

We conclude that the four constructs, including the toy promoter and 1,293 bp of surrounding DNA gave the expected expression pattern during embryonic as well as larval development. The putative Pax6-binding sites flanking the toy transcription start site might be of importance for initiation or maintenance of a correct expression pattern of the toy gene. Misexpression of Ey protein does not induce toy expression (Czerny et al. 1999). However, it is known that Pax6 genes in human and mouse are autoregulated (Plaza et al. 1993; Epstein et al. 1994; Aota et al. 2003) in order to keep the activated Pax6 gene continuously expressed also when the initiating signal is not longer present. How can UAS-toy be capable of rescuing lethal toy and ey mutants without a driver construct when toy expression seems to be so tightly regulated? Our results show that the UAS-toy transgene is leaky. Either very little Toy protein is required to rescue the pupal lethality of toy ${ }^{h d l}$ and the $h s p 70$ promoter included in the $p U A S T$ vector is sufficient to support this, or the UAS-toy transgene carries cryptic enhancers or auto-regulatory sequences. We hypothesize that Toy protein can bind to, e.g., the two putative Pax6-binding sites found in the toy transcription unit and thus maintain transcription of the toy gene. In the absence of Toy, Ey protein can bind to these sites and induce a low level of transcription. This may be sufficient for up-regulating UAS-toy transgene expression to rescuing levels, causing the observed leakiness of the transgenic lines. This hypothesis is supported by the fact the toy-Gal4 driver is necessary to obtain rescue with $U A S-P 35$.

Analyses of the published UAS-ey sequence (Halder et al. 1995) show that it does not contain any putative Pax6-binding sites. Still, a Gal4 driver is not necessary to increase survival or improve the eye phenotype of homozygous $e y^{D}$ adults; one copy of $U A S$-ey (or $U A S$ toy) is sufficient. This could be due to the fact that $e y^{D}$ is not a null allele, and the truncated protein can resume some functions. An increased copy number of wild-type toy or ey genes is then sufficient to ameliorate head development. The phenotypic improvement should, however, not be expected to be better than that seen in heterozygous $e y^{D}$ individuals, which is in agreement with our observations.

The D. melanogaster ey gene and vertebrate Pax6 genes have been shown to have binding sites for regulatory proteins within introns (Williams et al. 1998; Hauck et al. 1999; Kammandel et al. 1999; Adachi et al. 2003). Further analyses of presumptive toy enhancer regions are underway, especially focusing on the introns, to elucidate what signals initiate toy transcription in the eye-antennal primordia. toy mutant phenotypes and cell death in eye discs

Both Pax6 paralogs in Drosophila are necessary for correct development and survival. Furthermore, it is evident from their differential expression in brain and VNC that there has been a subdivision of functions between the toy and ey genes during Drosophila evolution. However, a plethora of evidence has accumulated implying that their functions in the eye development pathway are overlapping and redundant. To corroborate these results we further analyzed the phenotype of the toy ${ }^{h d l}$ mutant and investigated to what degree the two Pax6 transgenes could rescue these phenotypes.

Acridine orange staining shows a massive cell death in antennal discs of $t o y^{h d l}$ homozyogous mutant larvae and we also observe that antennal discs are missing in a large portion of the toy ${ }^{h d l}$ mutant larvae at third instar. In contrast, $e y^{D}$ mutant larvae mainly exhibit cell death in the eye disc, a phenotype very similar to that found in the $e y^{2}$ mutant (Halder et al. 1998). In larvae, there are indications that the adult eye development is regulated by the Notch (N) signaling pathway (Kumar and Moses 2001). It is possible to induce ectopic eyes with the constitutively activated UAS$\mathrm{N}^{\text {act }}$. This induces the expression of both ey and toy in the growing eye, indicating that $\mathrm{N}$ is upstream of toy (Kurata et al. 2000). Other studies, however, argue that $\mathrm{N}$ does not control ey expression or eye identity, but instead eye growth (Kenyon et al. 2003; Dominguez et al. 2004). We have previously shown that the pheno-critical period for producing the headless phenotype in toy ${ }^{\text {hdl }}$ mutants is during embryonic stages 12-16 (Kronhamn et al. 2002). It is, therefore, conceivable that the defects in eye-antennal discs of toy mutants are initiated when the small set of cells are allocated to form the eye-antennal primordium during gastrulation. If Toy is lacking, too few cells are set aside and this initial deficiency could be aggravated by apoptosis or a lower level of cell proliferation at later stages.

The weakest adult phenotype of toy ${ }^{h d l}$ mutants show lack of ocelli and cuticle derived from the ocellar part of the eye disc. A somewhat stronger phenotype is the "cleft head" where pharate adults lack substantial part of the dorsal medial head (Fig. 4f). These flies still retain the tissue derived from the antennal disc. An even stronger phenotype, "eye-on-thorax", is lacking antennal disc tissues, but still have compound eyes, which are positioned directly on the thorax. We sometimes observe that toy ${ }^{h d l}$ homozygous headless pharate adults have masses of red pigment inside the thorax (not shown). This indicates that compound eyes can be formed, but that they are unable to evaginate during metamorphosis, probably because head cuticle, on which the eyes should sit, is not formed. The strongest phenotype (headless) is pharate adults lacking all domains derived from the eye-antennal discs (Kronhamn et al. 2002). 
The deformation and cell death observed in the eyeantennal discs of toy mutants is clearly different from that in $e y^{D}$ and $e y^{2}$ mutants, where the eye part is strongly reduced in size and subject to massive cell death, while the antennal portion is normal (Halder et al. 1998). Cell death in $e y^{2}$ is very similar to that of $s o^{l}$ and $e y a^{l}$ (Bonini et al. 1993; Cheyette et al. 1994), two other genes specifying compound eyes. This shows that even if the expression patterns of ey and toy in early embryonic eye primordia and late larval eye-antennal imaginal discs are very similar (Czerny et al. 1999), the outcome of weaker mutations of the two Pax6 genes shows complementary phenotypes and is a good example of sub-functionality, which has also been pointed out by Gehring and co-workers (Punzo et al. 2004). While ey is vital for the development and differentiation of the compound eyes, toy is mainly needed for the development of the medial anterior, and dorsal parts of the head including antennae and ocelli.

\section{Rescue of ey mutants}

Misexpression of toy leads to the induction of ectopic eyes in an $e y^{2}$ as well as in an $e y^{J 5.71}$ mutant background (Punzo et al. 2004). We show that homozygous $e y^{J 5.71}$ loss of function mutant flies can be fully rescued by increased toy transcription, without any accompanying increase in ey expression. Thus, we conclude that Toy can promote eye and head development in an Ey-independent manner, which has also been suggested earlier (Punzo et al. 2004). The $\mathrm{Ey}^{\mathrm{D}}$ protein not only lacks the entire homeodomain, but also 660 amino acids in the $\mathrm{C}$-terminus, giving the dominant eyeless phenotype (Kronhamn et al. 2002). The dominance of the $e y^{D}$ allele is due to either a gain-of-function or a dominant-negative function of the $\mathrm{Ey}^{\mathrm{D}}$ protein. The remaining paired domain might still be able to bind to the cluster of binding sites within the eye enhancer of so, but the modifications in the C-terminus might interfere with normal transcriptional activation. We show that overexpression of either Ey or Toy proteins, respectively, can rescue the lethality of homozygous $e y^{D}$ mutants and also suppress the $e y^{D}$ phenotype and partially restore head development, and we have previously shown that the lethality could also be rescued by targeted expression of the apoptosis inhibitor P35 (Kronhamn et al. 2002). This seems to contradict a division of functions between the Pax6 paralogs in development, but a possibility is that the $E y^{D}$ protein (due to its conformational changes) cannot discriminate between Ey- and Toy-binding sites (for instance in so) and thus interferes with both developmental pathways. Such a scenario could also explain why $e y^{D}$ homozygous flies obtain a headless phenotype, whereas loss-of-function mutants do not.
Rescue from pupal lethality is caused by increased head size

The major lethal phase of headless mutants, toy ${ }^{\text {hdl }}$, toy ${ }^{G 739}$, and $e y^{D}$, is the pharate adult stage, when flies are ready to escape from the pupal case. One very important head structure for this process is the ptilinum, an extensible sac which is blown up by the pharate adult under hydrostatic pressure to break open the pupal lid. This structure is formed by the hinge region in the eye-antennal disc (Ouweneel 1970). This particular portion shows evidence of extensive cell death in toy ${ }^{\text {hdl }}$ mutants. A somewhat smaller head or an undeveloped ptilinum may make it impossible to break out from the pupa. We were able to partially rescue the three headless mutants from the pupal lethality by expressing Toy protein or Ey protein in eye primordia using UAS constructs. Also with the caspase inhibitor P35, we increased survival with similar efficiencies. This indicates that exogenous Pax6 proteins may have anti-apoptotic effects. Alternatively, ectopic expression of Toy or Ey induces proliferation and increases the number of cells set aside in the eye-antennal primordium during gastrulation resulting in an increased head size, which allows pharates to escape from pupal cases. A similar result could be achieved by P35-induced inhibition of apoptosis during later stages.

\section{Dosage sensitivity of Pax6}

In the toy ${ }^{\text {hdl }}$ mutant stock we observe an extreme temperature-dependent variability in the phenotypic manifestation (Kronhamn et al. 2002). One explanation for this could be a low affinity of wild-type Ey protein for Toy binding sites. At lower temperatures, the slow development allows Ey to bind in sufficient amounts to inhibit apoptosis and promote the developmental pathway. However, at higher temperatures, when development is rapid, not enough activation of target genes takes place, causing fewer founder cells and subsequent lethality. Interestingly, we find that it is only possible to rescue toy ${ }^{\text {hdl }}$ mutants from lethality when cultures are kept at lower temperatures $\left(15^{\circ}\right.$ to $\left.22^{\circ}\right)$. At $25^{\circ}$ we find that toy mRNA levels are elevated in toy ${ }^{\text {hdl }}$ flies carrying the UAS-toy construct and that this increase is independent of Gal4 driver constructs. Since the rescue at higher temperatures is very poor, we conclude that this increase in Toy expression levels results in lethality. Other publications also suggest that surplus of Pax6 protein function is detrimental to eye development. Over-expression of Ey protein in the eye discs leads to a reduced eye phenotype (Curtiss and Mlodzik 2000). Noll and co-workers (Jiao et al. 2001) have shown that over-expression of Pax proteins in the eye primordia results in interference of the developmental pathway and headless phenotypes. They also showed that this interference can only be induced 
during the first half of the embryonic development, when the eye-antennal primordium is formed and that the interference of the developmental pathway could be inhibited by ectopic expression of cell proliferation factors.

The vertebrate Pax6 homolog is involved in development of the vertebrate eye. Pax6 null alleles give a smalleye phenotype in heterozygous mice (Hill et al. 1992) and the human eye defect anririda (loss of iris) results from haplo-insufficiency of Pax6 (Ton et al. 1991). Homozygous Sey mice die at birth, lacking eyes and nose, and having severely malformed brains (Hogan et al. 1986). Similarly, rare incidents of compound heterozygotes for Pax6 mutations in humans result in lethality; including lack of eyes, a malformed nasal region, and severe brain defects, where the cerebral hemispheres are widely separated (Glaser et al. 1994). This shows that Pax6 in vertebrates, like in Drosophila, is extremely dosage-sensitive and that heterozygous mutants show a phenotype similar to ey mutants, while the homozygous phenotype resemble the toy mutant phenotype. Thus, it seems as the two Drosophila Pax6 genes, toy and ey, have acquired a certain degree of subfunctionality during evolution, and the two copies together reflect the functions of the single Pax6 gene in vertebrates.

Acknowledgments We are grateful to Per Hörstedt for scanning electron microscopy, Ingrid Dacklin, Umeå Fly and Worm Transgene Facility, for generation of transgenic Drosophila stocks, Kerstin Kristiansson for technical assistance, Erich Frei and Markus Noll for valuable advice and fly stocks. We also thank Andrew Lambertsson and the Bloomington Stock Center for fly stocks. This work has been supported by the Swedish Research Council, J.C. Kempe Memorial Fund, Sven and Lilly Lawski Foundation and the Royal Physiographic Society.

Open Access This article is distributed under the terms of the Creative Commons Attribution Noncommercial License which permits any noncommercial use, distribution, and reproduction in any medium, provided the original author(s) and source are credited.

\section{References}

Adachi Y et al (2003) Conserved cis-regulatory modules mediate complex neural expression patterns of the eyeless gene in the Drosophila brain. Mech Dev 120:1113-1126

Aota S, Nakajima N, Sakamoto R, Watanabe S, Ibaraki N, Okazaki K (2003) Pax6 autoregulation mediated by direct interaction of Pax6 protein with the head surface ectoderm-specific enhancer of the mouse Pax6 gene. Dev Biol 257:1-13

Bonini NM, Leiserson WM, Benzer S (1993) The eyes absent gene: genetic control of cell survival and differentiation in the developing Drosophila eye. Cell 72:379-395

Bonini NM, Bui QT, Gray-Board GL, Warrick JM (1997) The Drosophila eyes absent gene directs ectopic eye formation in a pathway conserved between flies and vertebrates. Development 124:4819-4826

Brand AH, Perrimon N (1993) Targeted gene expression as a means of altering cell fates and generating dominant phenotypes. Development 118:401-415
Brand AH, Perrimon N (1994) Raf acts downstream of the EGF receptor to determine dorsoventral polarity during Drosophila oogenesis. Genes Dev 8:629-639

Breitling R, Gerber JK (2000) Origin of the paired domain. Dev Genes Evol 210:644-650

Callaerts PC, Francis J, Hens CK (2006) Pax6 and eye development in Arthropoda. Arthropod Struct Dev 35:379-391

Cheyette BN, Green PJ, Martin K, Garren H, Hartenstein V, Zipursky SL (1994) The Drosophila sine oculis locus encodes a homeodomain-containing protein required for the development of the entire visual system. Neuron 12:977-996

Chi N, Epstein JA (2002) Getting your Pax straight: Pax proteins in development and disease. Trends Genet 18:41-47

Curtiss J, Mlodzik M (2000) Morphogenetic furrow initiation and progression during eye development in Drosophila: the roles of decapentaplegic, hedgehog and eyes absent. Development 127:1325-1336

Czerny T, Halder G, Kloter U, Souabni A, Gehring WJ, Busslinger M (1999) twin of eyeless, a second Pax-6 gene of Drosophila, acts upstream of eyeless in the control of eye development. Mol Cell 3:297-307

Dominguez M, Ferres-Marco D, Gutierrez-Avino FJ, Speicher SA, Beneyto M (2004) Growth and specification of the eye are controlled independently by Eyegone and Eyeless in Drosophila melanogaster. Nat Genet 36:31-39

Enerly E, Larsson J, Lambertsson A (2003) Silencing the Drosophila ribosomal protein L14 gene using targeted RNA interference causes distinct somatic anomalies. Gene 320:41-48

Epstein J, Cai J, Glaser T, Jepeal L, Maas R (1994) Identification of a Pax paired domain recognition sequence and evidence for DNAdependent conformational changes. J Biol Chem 269:8355-8361

Finkelstein R, Perrimon N (1991) The molecular genetics of head development in Drosophila melanogaster. Development 112:899-912

Garcia-Bellido A, Merriam JR (1969) Cell lineage of the imaginal discs in Drosophila gynandromorphs. J Exp Zool 170:61-75

Glaser T, Jepeal L, Edwards JG, Young SR, Favor J, Maas RL (1994) PAX6 gene dosage effect in a family with congenital cataracts, aniridia, anophthalmia and central nervous system defects. Nat Genet 7:463-471

Halder G, Callaerts P, Gehring WJ (1995) Induction of ectopic eyes by targeted expression of the eyeless gene in Drosophila. Science 267:1788-1792

Halder G, Callaerts P, Flister S, Walldorf U, Kloter U, Gehring WJ (1998) Eyeless initiates the expression of both sine oculis and eyes absent during Drosophila compound eye development. Development 125:2181-2191

Hauck B, Gehring WJ, Walldorf U (1999) Functional analysis of an eye specific enhancer of the eyeless gene in Drosophila. Proc Natl Acad Sci USA 96:564-569

Hay BA, Wolff T, Rubin GM (1994) Expression of baculovirus P35 prevents cell death in Drosophila. Development 120:2121-2129

Hill RE et al (1992) Mouse Small eye results from mutations in a paired-like homeobox-containing gene. Nature 355:750

Hogan BL, Horsburgh G, Cohen J, Hetherington CM, Fisher G, Lyon MF (1986) Small eyes (Sey): a homozygous lethal mutation on chromosome 2 which affects the differentiation of both lens and nasal placodes in the mouse. J Embryol Exp Morphol 97:95-110

Jang CC et al (2003) Two Pax genes, eye gone and eyeless, act cooperatively in promoting Drosophila eye development. Development 130:2939-2951

Jarrett HW (2000) Temperature dependence of DNA affinity chromatography of transcription factors. Anal Biochem 279:209-217

Jaworski C, Sperbeck S, Grahamn C, Wistow G (1997) Alternative splicing of Pax6 in bovine eye and evolutionary conservation of intron sequences. Biochem Biophys Res Commun 240:196-202 
Jiao R, Daube M, Duan H, Zou Y, Frei E, Noll M (2001) Headless flies generated by developmental pathway interference. Development 128:3307-3319

Kammandel B, Chowdhury K, Stoykova A, Aparicio S, Brenner S, Gruss P (1999) Distinct cis-essential modules direct the timespace pattern of the Pax6 gene activity. Dev Biol 205:79-97

Kammermeier L et al (2001) Differential expression and function of the Drosophila Pax6 genes eyeless and twin of eyeless in embryonic central nervous system development. Mech Dev 103:71-78

Kenyon KL, Ranade SS, Curtiss J, Mlodzik M, Pignoni F (2003) Coordinating proliferation and tissue specification to promote regional identity in the Drosophila head. Dev Cell 5:403-414

Kronhamn J et al (2002) Headless flies produced by mutations in the paralogous Pax6 genes eyeless and twin of eyeless. Development 129:1015-1026

Kumar JP, Moses K (2001) EGF receptor and Notch signaling act upstream of eyeless/Pax6 to control eye specification. Cell 104:687-697

Kurata S, Go MJ, Artavanis-Tsakonas S, Gehring WJ (2000) Notch signaling and the determination of appendage identity. Proc Natl Acad Sci USA 97:2117-2122

Lenhard B, Sandelin A, Mendoza L, Engstrom P, Jareborg N, Wasserman WW (2003) Identification of conserved regulatory elements by comparative genome analysis. J Biol 2:13

Miller DJ et al (2000) Pax gene diversity in the basal cnidarian Acropora millepora (Cnidaria, Anthozoa): implications for the evolution of the Pax gene family. Proc Natl Acad Sci USA 97:4475-4480

Ouweneel WJ (1970) Normal and abnormal determination in the imaginal discs of Drosophila, with special reference to the eye discs. Acta Embryol Exp (Palermo) 1:95-119

Pan D, Rubin GM (1998) Targeted expression of teashirt induces ectopic eyes in Drosophila. Proc Natl Acad Sci USA 95:1550815512

Patel NH (ed) (1994) Imaging neuronal subsets and other cell types in whole-mount Drosophila embryos and larvae using antibody brobes. Academic press, San Diego
Plaza S, Dozier C, Saule S (1993) Quail Pax-6 (Pax-QNR) encodes a transcription factor able to bind and trans-activate its own promoter. Cell Growth Differ 4:1041-1050

Punzo C, Kurata S, Gehring WJ (2001) The eyeless homeodomain is dispensable for eye development in Drosophila. Genes Dev $15: 1716-1723$

Punzo C, Seimiya M, Flister S, Gehring WJ, Plaza S (2002) Differential interactions of eyeless and twin of eyeless with the sine oculis enhancer. Development 129:625-634

Punzo C et al (2004) Functional divergence between eyeless and twin of eyeless in Drosophila melanogaster. Development 131:39433953

Seimiya M, Gehring WJ (2000) The Drosophila homeobox gene optix is capable of inducing ectopic eyes by an eyeless-independent mechanism. Development 127:1879-1886

Shen W, Mardon G (1997) Ectopic eye development in Drosophila induced by directed dachshund expression. Development 124:4552

Ton CC et al (1991) Positional cloning and characterization of a paired box- and homeobox-containing gene from the aniridia region. Cell 67:1059-1074

Williams SC, Altmann CR, Chow RL, Hemmati-Brivanlou A, Lang RA (1998) A highly conserved lens transcriptional control element from the Pax-6 gene. Mech Dev 73:225-229

Wolff T (2000) Acridine orange. In: Sullivan W, Ashburner M, Hawley RS (eds) Drosophila protocols. CSHL Press, New York, pp 221222

Zhang Y, Emmons SW (1995) Specification of sense-organ identity by a Caenorhabditis elegans Pax-6 homologue. Nature 377:55-59

Zhang T, Ranade S, Cai CQ, Clouser C, Pignoni F (2006) Direct control of neurogenesis by selector factors in the fly eye: regulation of atonal by Ey and So. Development 133:4881-4889 